Joerg Subke*, Benedict Schneider, Fiona Hanitz, Sabine Krüger, Heinz-Otto Junker, Hans-Joachim Schwalbe and Udo Wolf

\title{
Clinical case study in acoustic-kinetic joint analysis: Synchronization and Evaluation of kinetic measurement data in AEA (Acoustic Emission Analysis) based diagnosis of arthritic knee joint defects
}

\begin{abstract}
In patients with arthritic knee joint defects the course of movement, the application of muscle forces and the degree of freedom of the joints of the lower limbs differs significantly from the corresponding data of a healthy proband. The enhanced acoustic-kinetic joint analysis based on AEA, ground force reaction measurements and video enhanced gait analysis permits the correlation of force data, joint angles and acoustic emission significant of defective joint cartilage regions. This diagnostic procedure permits a quantifiable and detailed non invasive diagnosis of lesion patterns in the arthritic knee joint by means of a synchronization algorithm. The AEA shows lesion signals in the first and third knee bend of the patient. The lesion signals are assigned to the joint angles and ground reaction force. Comparing the kinematical data there is a shift between the curves of the hip and the knee angle. Overall the force data represent an imbalance between the left and the right leg during the knee bends. The presentation of the three measurement systems, the method, the synchronization of the data sets and their final assessment as well as the occurring difficulties during a case of a clinical study are discussed. This would be helpful regarding further patients of the clinical study.
\end{abstract}

Keywords: Acoustic emission analysis, acoustic-kinetic analysis, arthrosis, knee joint defect, dynamic analysis, diagnosis.

https://doi.org/10.1515/cdbme-2021-2203

\section{Introduction}

A diagnostic system based on Acoustic Emission Analysis, combined with the measurement of ground reaction forces and video gait analysis, aims at a non-invasive dynamic assessment of knee joint defects. In addition to classical diagnostic routines this system provides a range of data on lesion patterns and the kinematic and biomechanical characteristics of patients impeded by arthrosis of the knee joint $[1,2]$.

In cooperation with the Rheumazentrum Mittelhessen in Bad Endbach, a competence centre for rheumatology and degenerative joint diseases, a first clinical case study on the acoustic - kinetic joint analysis was put into practice and the results of the patient were quantified and discussed.

\section{Material \& Method}

The acoustic-kinetic joint analysis comprises:

\footnotetext{
*Corresponding author: Joerg Subke: University of Applied Sciences THM, Wiesenstr. 14, Giessen, Germany, e-mail: joerg.subke@Ise.thm.de

Benedict Schneider, Fiona Hanitz: University of Applied Sciences THM, Giessen, Germany

Sabine Krüger: Osteopathiepraxis, Ludwigsburg, Germany Heinz-Otto Junker: Rheumazentrum, Bad Endbach, Germany Hans-Joachim Schwalbe: BoneDias, Greifenstein, Germany Udo Wolf: University of Applied Sciences, Fulda, Germany
}

- Video based gait analysis with Tracker ${ }^{\circledR 5}$.1.5 and two cameras (Basler Scout scA640-120gm) recording frontal and sagittal view of the patient during a $10 \mathrm{sec}$. period of standard movement

- Measurement of ground reaction forces and force balance by Zebris ${ }^{\circledR} F D M ~ S ~(2560$ sensors, $120 \mathrm{~Hz}$, range 1-120 $\mathrm{N} / \mathrm{cm}^{2}$, accuracy $\pm 5 \%$, resolution 1,4 sensors $/ \mathrm{cm}^{2}$ ) 
- Acoustic Emission Analysis BoneDiaS ${ }^{\circledR}$ (mobile system, telemetric measurement time $10 \mathrm{sec})[3,4]$

The measurement is aiming at objective data on divergent behavior in the patient's motion pattern, the mobility of the joints and at information about the existence, localization and extend of cartilage damage. Patients with arthrosis develop strategies to keep the symptoms manageable, so if they cannot avoid a movement that will cause pain, they will e.g. shift load balance and in the long run, cause new sets of symptoms. The complex acoustic-kinetic joint analysis attempts to detect these strategies and to qualify and quantify the causative disease patterns.

The measurement systems have to be started manually and separately. As yet there is no possibility to connect and synchronize them automatically. On the synchronization and analysis of the data sets see a prior publication [5].

The exemplary patient in this case study is female, 68 years old, has a body mass of $60 \mathrm{~kg}$ and height of $163 \mathrm{~cm}$. As she suffers from pains in the left knee joint that occur occasionally during routine movements and frequently under physical strain, sports, gymnastics, arthrosis of the left knee joint is suspected.

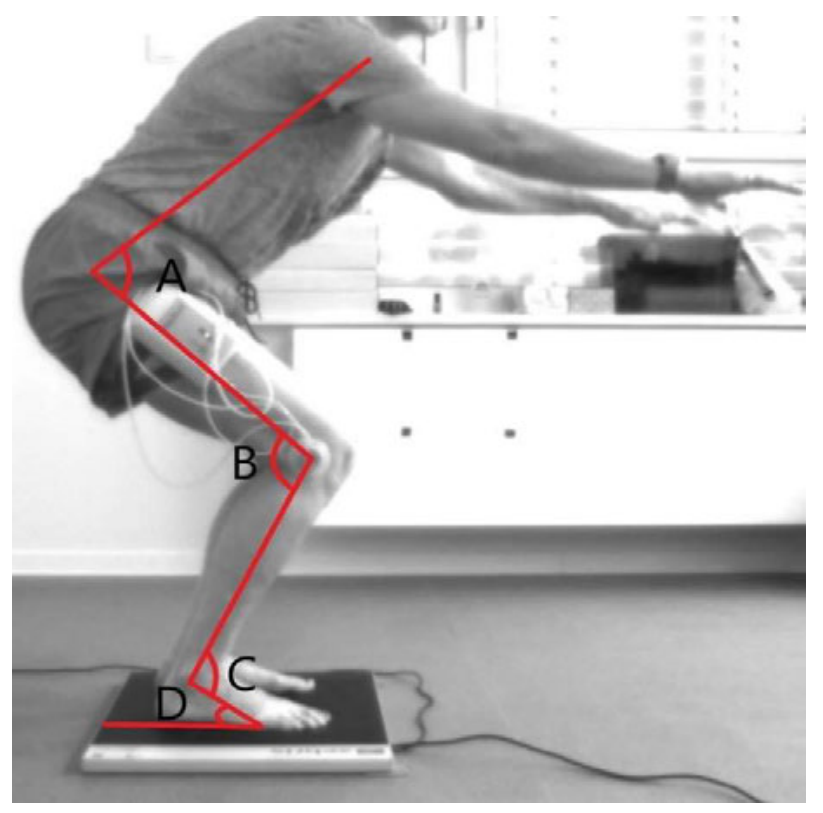

Figure 1: Sagittal angles analysed in Tracker. (A) hip angle; (B) knee joint angle; (C) ankle joint angle; (D) ankle-floor angle

The patient performs the standard movement on the Zebris ${ }^{\circledR}$ plate. The standard movement consists of a series of three knee bends, flexion of knee joint to the maximum of the patient's capacities, overall measurement time $10 \mathrm{sec}$. The AEA sensor is adapted first on the patient's left condyle, in further measurements on the right. Fig. 1 represents the sagittal view from the video analysis system with the joint angles.

\section{Results and Discussion}

AEA enables us to observe the dynamic processes in the knee joint. Damage or wear to the cartilage as well as lesions emit characteristic acoustic signals according to their intensity. The acoustic signals can be classified into 4 categories (table 1):

Table 1: AEA Signal Categories

\begin{tabular}{|c|c|c|}
\hline Category & Description & Color \\
\hline 1 & Roughness & Green \\
\hline 2 & Light lesions & Blue \\
\hline 3 & $\begin{array}{l}\text { Lesions near the } \\
\text { arthritic case }\end{array}$ & Red \\
\hline 4 & Arthritic case & Black \\
\hline
\end{tabular}

In the graphical representation, color values are assigned to these categories (Table 1). In Fig. 7 and Fig. 8, the colored vertical lines indicate at which point in time and at which force-angle environment the AEA detects anomalies and the intensity or class/category/degree of damage.

In the present case, AEA signals pointed to cartilage damage in both knees. While there were category 1 signals in the right knee that indicate a mere roughness, the left knee emitted 4 signals of varying intensity. Three signals of the categories 1 (L2 in Fig. 3), 3 (L1 in Fig. 2) and 4 (L3 in Fig. 4) during the first knee bend and one category 1 signal in the course of the third knee bend (L4 in Fig. 5).

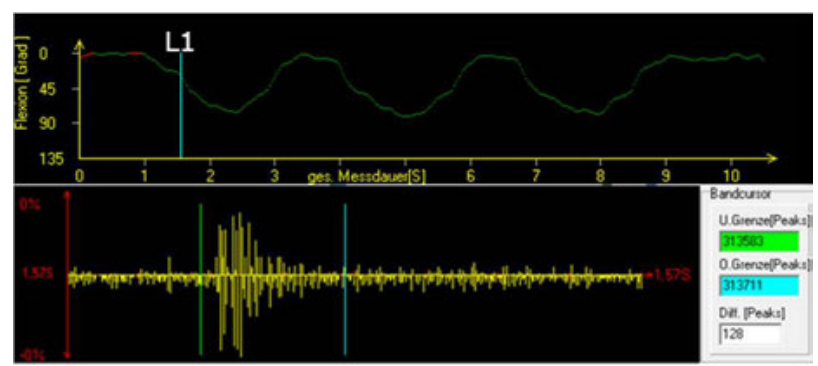

Figure 2: a) Angle of the three knee bends, blue location of the signal $L 1 ; b) A E$ of the signal $L 1$; Lesion near arthritic case (category 3) 


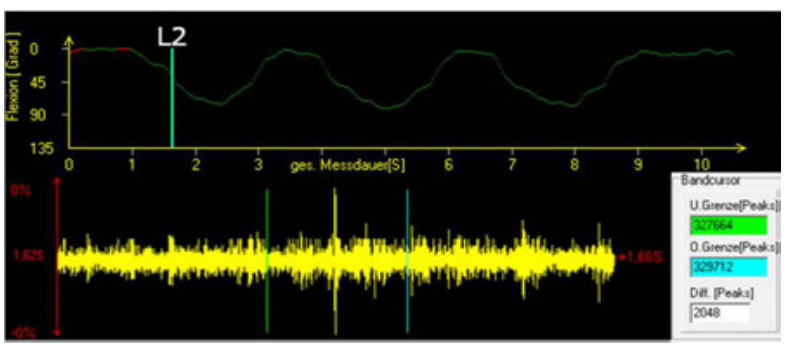

Figure 3: a) Angle of the three knee bends, blue location of signal L2; b) AE of Signal L2; roughness (category 1)

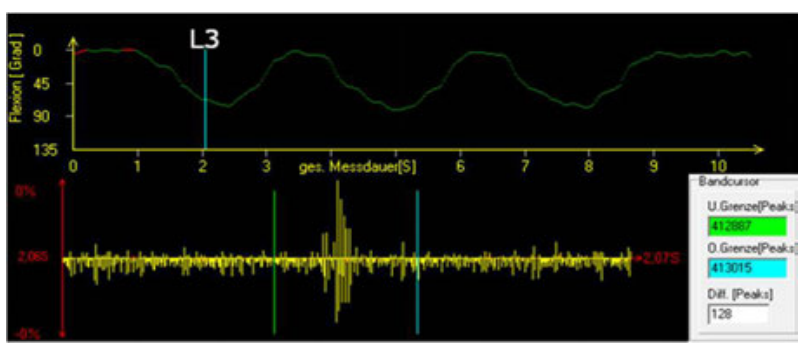

Figure 4: a) Angle of the three knee bends, blue location of the signal L3; b) AE of the signal L3; arthritic case (category 4)

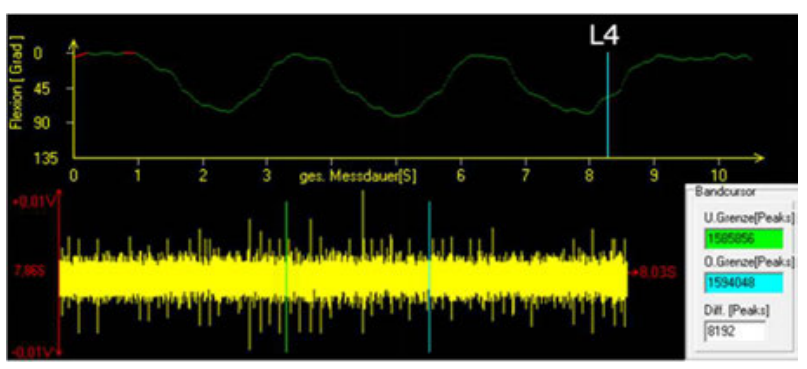

Figure 5: a) Angle of the three knee bends, blue location of the signal L4; b) AE of the signal L4; roughness (category 1)

The fact that the AEA signals of the first knee bend are not repeated in the second and third shows that the patient uses different contact paths between tibia and femur. In addition it is an indication that not (yet) all of the cartilage surface is damaged. Comparing the first and third knee bend a category 1 signal (roughness) can be detected at a similar knee joint angle as the category 4 (arthrosis) signal that showed in the first knee bend. This roughness indicates the border of the category 4 signal.

In addition to the acoustic emission of the knee joint during the series of movements the total muscle force, the forces of the left and right leg and the difference between the ground forces in the left and right leg (L-R) were analyzed. And the joint angle values as introduced in Fig. 1.

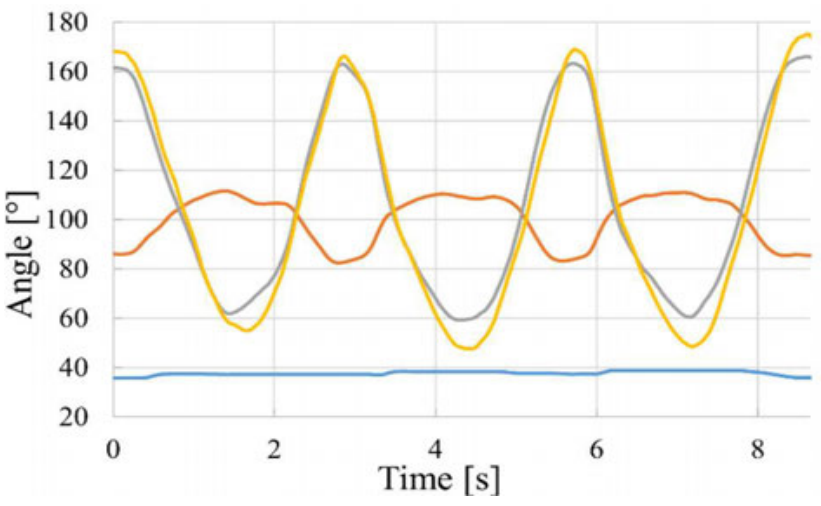

Figure 6: Sagittal joint angle analysis. Blue ankle-to-floor angle. Orange ankle joint angle. Grey knee joint angle. Yellow hip joint angle.

Fig. 6 shows the graphical representation of an automatically synchronized data set from the video based gait analysis. In the unimpeded movement of a healthy proband, the maxima of the hip, knee and ankle angles would occur at the same time. In this case, the maximum values of hip (yellow) and knee (grey) joint angles are out of alignment. This is very obvious during the first knee bend, a little less in the second and in the third, as the patient has successfully changed the load path the maxima are approximately simultaneous.

The AEA data include measurements of an angle that observes the position of the "toolbox" fixed to the patient's thigh in relation to gravity (Fig. 1). Therefore it is possible to match angles at the beginning of the patient's motion sequence, when the joint values are identical and thus synchronize the measurements [5].

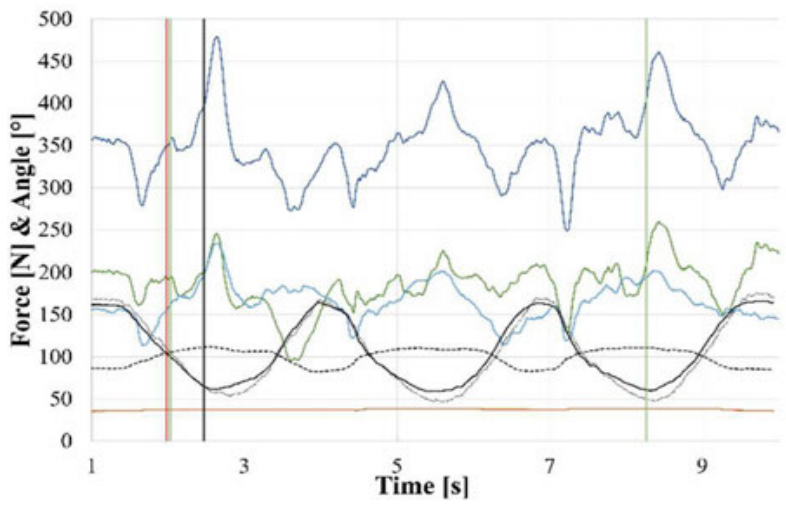

Figure 7: Force and joint angle curves with AE Signals L1-L4 coloured to their severity according to Table 1. Dark blue total force, green left leg force, light blue right leg force, black dotted hip joint angle, black knee joint angle, black broken ankle angle, orange ankle-floor angle. Vertical lines see Table 1. 
By adding the total force to the picture (Fig. 7) a graphical representation of the synchronized data sets is obtained. The total force (blue) shows that the patient executed her three knee bends without pausing at the lowest point of the bend. The variability in the execution of the knee bends is high but healthy probands tend to pause and create a local minimum in the acting force. The patient's dominant leg is the left, but during the first knee bend of the knee, she shifts the loads from left to right, probably experiencing or expecting pain. And this is where we observe AE signals indicating damage of various intensity (vertical lines) in the course of the downward movement. During knee bend 2 and 3, she shifts her load balance back to the dominant leg, but she now uses a different load path to avoid the lesioned areas of cartilage.

Comparing the course of the hip and knee joint angles, there is not an isochronous development of the two angles. This indicates that the patient stopped the flexion of the knee joint earlier than the flexion of the hip joint which indicates a strategy to relieve the left knee.

A closer analysis of the muscle loads of the left and right leg $(L-R)$ reveals again the load accent on the left leg except during the first knee bend where the patient according to the vertical lines of the AEA put strain on the lesions in her joint (Fig. 8).

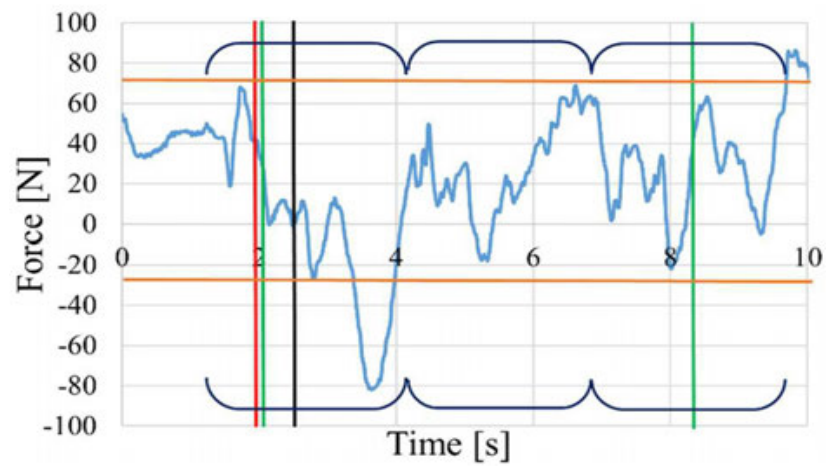

Figure 8: Force emitted by right leg subtracted from force emitted by left leg (L-R). Brackets show the knee bends. Blue line force distribution. Orange lines are usual range of force distribution. Red, green, black lines are AE Signals coloured according to severity shown in Table 1.

\section{Conclusion}

The Acoustic Emission Analysis is the only non-destructive method to assess the condition of joint cartilage in a dynamic process. Coupled with Gait Analysis and Muscle Force data, the acoustic kinetic data sets provide extensive information on the patient's condition and the resulting strategies to relieve the symptoms of arthritic defects and possible subsequent complaints.

In the presented case roughened areas were found in the patient's right knee and in the left knee various stages of alterations from roughness and lesions to loss of cartilage. The AEA results confirm the clinical assumption that the left knee is more seriously affected than the right.

The results lead to the recommendation that the patient should receive physiotherapy in order to alter her dominant side from the left to the right. In addition she should wear orthopedic insoles and thus effectively reroute the contact path away from the arthritic area. This would slow the further deterioration of the affected cartilage in tibia and femur.

\section{Author Statement}

Research funding: The author state no funding involved. Conflict of interest: Authors state no conflict of interest. Informed consent: Informed consent has been obtained from all individuals included in this study. Ethical approval: The research related to human use complies with all the relevant national regulations, institutional policies and was performed in accordance with the tenets of the Helsinki Declaration, and has been approved by the authors' institutional review board or equivalent committee.

\section{References}

[1] Kiselev J, Wolf U, Ziegler B, Schwalbe H-J, Franke R-P. Detection of early phases of ostearthritis using acoustic emission analysis. Med. Eng. Phys. 2019, 65, 57-60.

[2] Subke J, Krueger S, Junker HO, Schwalbe HJ, Franke RP, Wolf U. An introduction to Acoustic Emission Analysis (AEA) based medical diagnosis techniques: Screening and Monitoring of cartilage defects in knee osteoarthrosis. Current Directions in Biomedical Engineering 2019; 5(1):1-3)

[3] Schwalbe HJ, Schmale J, Subke J, Ziegler B, Kellotat A, Dörner P, Franke RP, Wolf U. Detection of Defects in the Human Skeletal System and Production of Failure Optimized Artificial Bone Applying Acoustic Emission Analysis (AEA). $31^{\text {st }}$ Conference of the European Working Group on Acoustic Emission (EWGAE) Proceedings, Dresden 2014

[4] Franke RP, Schwalbe HJ, Kiselev J, Wolf U, Subke J, Ziegler B. Schallemissionsanalyse zum Nachweis von Gelenkdefekten in der medizinischen Diagnostik. Deutsche Gesellschaft für zerstörungsfreie Prüfung 18. Kolloquium Schallemission, Berichtsband 2011

[5] Subke J, Schneider B, Hanitz F, Wolf U. Acoustic-kinetic joint analysis: Synchronization and Evaluation of kinetic measurement data in AEA (Acoustic Emission Analysis) based diagnosis of arthritic knee joint defects. Current Directions in Biomedical Engineering 2021 